\title{
Mild androgen insensitivity syndrome presenting as male infertility with azoospermia
}

Dharshini Karuppiah', Sanduni Kudabadu ${ }^{1}$

${ }^{1}$ Diabetes and Endocrine unit, Teaching Hospital, Batticaloa

\section{Abstract}

Androgen insensitivity syndrome results from a mutation in androgen receptor gene. It has wide spectrum of phenotypic presentations. Mild androgen insensitivity syndrome (MAIS) is the milder form, present as undervirilized male syndrome. In some instances, the only observed abnormality appears to be male infertility; therefore, MAIS could explain some cases of idiopathic male infertility. Here we describe a case of MAIS in a 38 years old male who presented with azoospermia and infertility.

DOI: http://doi.org/10.4038/sjdem.v8i1.7352

Correspondence: e-mail: kdharshinik@gmail.com

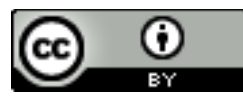

This is an open-access article distributed under the terms of the Creative Commons Attribution 4.0 International License, which permits unrestricted use, distribution and reproduction in any medium provided the original author and source are credited 


\section{Introduction}

Androgen insensitivity syndrome (AIS) is typically characterized by evidence of under-masculization of the external genitalia at birth, abnormal secondary sexual development in puberty and infertility in individuals with a 46 XY karyotype (1). AIS results from a mutation in androgen receptor gene (Xq11-12). It is an inherited in an $\mathrm{X}$ linked recessive fashion, but $40 \%$ patients have negative family history. Incidence is estimated to be 1:20000-1:64000 male births (2). AIS can be subdivided in to 3 broad phenotypes: complete androgen insensitivity syndrome (CAIS) with typical female genitalia, partial androgen insensitivity (PAIS) with predominantly female, predominantly male or ambigous genitalia; mild androgen insensitivity syndrome (MAIS) with typical male genitalia. MAIS is the rarest form and diagnosis may be delayed or even missed. This could explain some cases of male infertility. Here we describe a case of MAIS in a 38 years old male who presented with azoospermia and infertility.

\section{Case}

A 38-year old man was referred to the Endocrinology department of Teaching Hospital Batticaloa for evaluation of subfertility. He was married for 3 years and unsuccessful in attempting to father a pregnancy. He had no significant medical or surgical history, was not on any long-term medications. He did not smoke or consumes alcohol. He was short with a height of 146 $\mathrm{cm}$ and weighing $45.5 \mathrm{~kg}$. He was under-virilized with reduced body hair, but he had male pattern facial hair and there was no gynaecomastia. Genital examination revealed low testicular volume of $4 \mathrm{ml}$, penile shaft of $7 \mathrm{~cm}$ and Tanner stage iii pubic hair distribution (figure 1).

Semen analysis performed on several occasions revealed very low volume ejaculate with azoospermia. His testosterone level drawn at 8 am was $6.132 \mathrm{ng} / \mathrm{ml}$ (normal, $3.4-8.7 \mathrm{ng} / \mathrm{mL}$ ). Repeat morning serum testosterone done at medical research institute was $27.62 \mathrm{nmol} / 1$ (normal, $8.4-28.7 \mathrm{nmol} / \mathrm{l}$ ). Serum luteinizing hormone $(\mathrm{LH})$ and follicle-stimulating hormone (FSH) were elevated and prolactin levels were within the normal range (table 1). Transrectal sonographic imaging of the prostate and surrounding structures revealed no evidence of ejaculatory duct obstruction or other abnormality. His karyotyping was 46XY.

The patient's wife was a 31-year-old woman with no known fertility concerns. She had regular menses, and her past medical history was negative for any surgical or medical conditions that might affect her fertility status.

\section{Table 1: Hormone evaluation of the patient.}

\begin{tabular}{llc} 
Tests & Results & Normal Range \\
\hline TSH (mIU/L) & 1.96 & $0.46-4.68$ \\
Free T4 (pmol/L) & 14.5 & $10.0-28.2$ \\
LH (mIU/L) & 9.01 & $1.31-10.50$ \\
FSH (mIU/L) & 26.3 & $1.55-9.74$ \\
Prolactin (mIU/L) & 357 & $78-380$ \\
Testosterone (ng/ml) & 6.132 & $2.4-8.7$ \\
$\quad(\mathbf{n m o l} / \mathbf{l})$ & 27.62 & $8.4-28.7$
\end{tabular}

*TSH - thyroid stimulating hormone; LH - luteinizing hormone; FSH - follicular stimulating hormone 


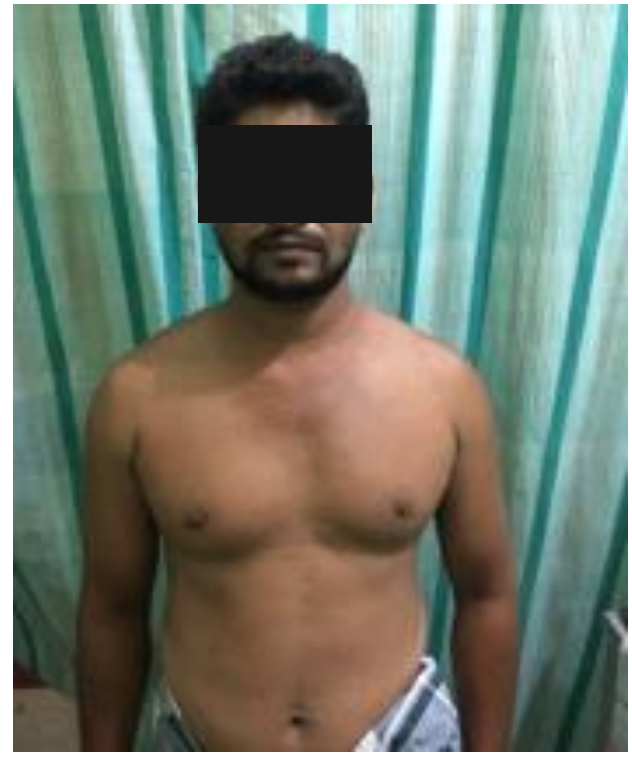

Figure 1: The patient with reduced body hair.

\section{Discussion}

Androgen insensitivity syndrome was first described by J.M.Morris an American gynaecologist in 1953(3). The basic etiology is a loss of function mutation in the androgen receptor (AR) gene, which has been localized to the long arm of $\mathrm{X}$ chromosome. Over 1000 such mutations have been described, including complete and partial gene deletions, point mutations and small deletions (4). Androgen insensitivity syndrome represents a spectrum of defects in androgen action and can be subdivided in to 3 phenotypes; a. Complete androgen insensitivity syndrome (CAIS) with typical female genitalia, b. Partial androgen insensitivity syndrome (PAIS) with predominantly female, predominantly male, or ambiguous genitalia and c. Mild androgen insensitivity syndrome (MAIS) with typical male genitalia.

There are no formal diagnostic criteria available due to large variance seen at the molecular, biochemical, and morphologic levels with the various AIS phenotypes. But AIS should be suspected in an individual with a 46 XY karyotype who has following characteristics; undermasculinization of the external genitalia, impaired spermatogenesis with otherwise normal testes, absent or rudimentary mullerian structures, evidence of normal or increased synthesis of testosterone and its normal or increased luteinizing hormone and/ or by the

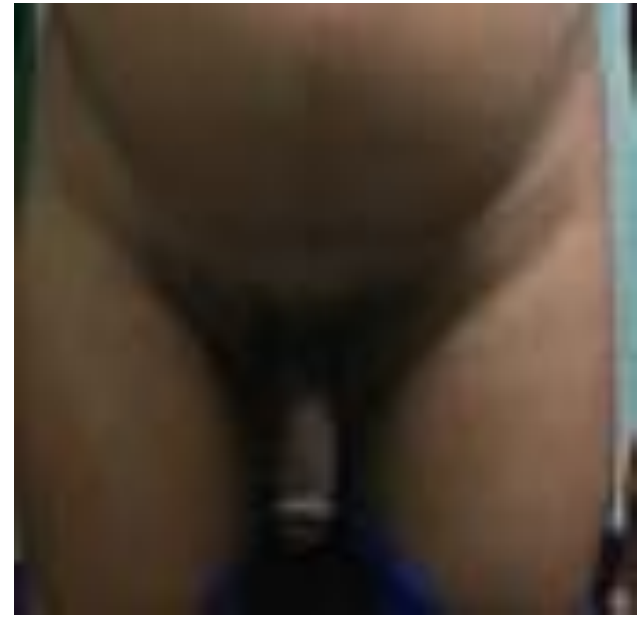

Figure 2: The patient with undervirilized external genitalia.

identification of a hemizygous pathogenic variant in $A R$ (1).

MAIS, present as undervirilized male syndrome. The external genitalia of affected individuals are unambiguously male. They usually present with gynecomastia at puberty. They may have undermasculinization that includes sparse facial and body hair and small penis. Erectile dysfunction may be a complaint. Spermatogenesis may or may not be impaired. In some instances, the only observed abnormality appears to be male infertility; therefore, MAIS could explain some cases of idiopathic male infertility (1). MAIS caused by singlenucleotide variants of $A R(5)$ may be clinically indistinguishable from MAIS caused by expansion of the polymorphic $\mathrm{CAG}$ repeat in $A \mathrm{R}(6)$. Pathogenic expansion of this triplet repeat is the cause of spinal and bulbar muscular atrophy, also known as Kennedy disease. Some cancers show somatic alterations in $A R$. These, however, appear to result in a gain of function rather than the loss of function seen in AIS.

The present case is compatible with MAIS, though the mutation in $A R$ gene was not confirmed. MAIS is a condition that results in a mild impairment of the cell's ability to respond to androgens. The degree of impairment is sufficient to impair spermatogenesis and/or the development of secondary sexual characteristics at 
puberty in males but doesn't affect genital differentiation or development. Female genital and sexual development is not significant affected by the insensitivity to androgens; as such, MAIS is only diagnosed in males (7).

MAIS has a mild presentation that often goes unnoticed and untreated. Similarly, our patient had features of under-virilization, but he did not seek any medical attention. He was investigated for subfertility at the age of 38 and found to have azoospermia.

Management of MAIS is currently limited to symptomatic management. This includes surgical correction of mild gynaecomastia, and minor hypospadias repair. Patients may be treated with testosterone or dihydrotestosterone (DHT). The advantage of DHT is that it cannot be aromatized to estrogen. No medical consensus has been reached about this therapy, and no dosage schedules have been established (4). High dose testosterone therapy has been shown to improve virilization in men with MAIS (7). Managing infertility in MAIS is difficult when spermatogenesis is severely impaired. Normal spermatogenesis is dependent on appropriate signaling from the hypothalamic pituitary gonadal axis. The use of exogenous androgens can influence the axis by exerting negative feedback, and ultimately decrease or complete cessation of spermatogenesis. But, there are reports of high dose testosterone therapy associated with reversing infertility due to low sperm count (8). In addition, psychological support is probably the most important aspect of medical care.

Our patient declined high dose testosterone treatment. Following discussion, he and his wife decided for assisted reproduction with sperm donation.

\section{Conclusion}

MAIS often goes unnoticed and untreated due to its mild presentation. Patient will be benefited by testosterone supplementation and other symptomatic management. This case sheds light on the need for screening for mild androgen insensitivity when a patient presents with unexplained infertility and mild hypoandrogenic features.

\section{References}

1. Gottlieb B, Trifiro MA. Androgen Insensitivity Syndrome. Gene Reviews. 1999 Mar. https://www.ncbi.nlm.nih.gov/books/NBK1429.

2. Kour V, Abrol A. Testicular Feminization Syndrome. JK Science 2005; 7(1).

3. Morris JM. The syndrome of testicular feminization in male pseudohermaphrodite. Am J Obstet Gynecol 1953: 65(6):1192-1211.

4. Christian A Koch, Medscape. Updated: Oct 16, 2017.

5. Wang Q, Ghadessy FJ, Yong EL. Analysis of the transactivation domain of the androgen receptor in patients with male infertility. Clinical Genetics. 1998 Sep; 54(3): 185-192.

6. Tut TG, Ghadessy FJ, Trifiro MA, Pinsky L, Yong EL. Long polyglutamine tracts in the androgen receptor are associated with reduced transactivation, impaired sperm production, and male infertility. J Clin Endocrinol Metab. 1997; 82:3777-3782.

7. Quigley CA, De Bellis A, Marschke KB, el-Awady MK, Wilson EM, French FS. Androgen receptor defects: historical, clinical, and molecular perspectives. Endocr. Rev. 1995 June;16 (3): 271-321. doi:10.1210/edrv-16-3-271

8. K. M. Tordjman, M. Yaron, A. Berkovitz, A. Botchan, C. Sultan, S. Lumbroso. Fertility after high-dose testosterone and intracytoplasmic sperm injection in a patient with androgen insensitivity syndrome with a previously unreported androgen receptor mutation. Andrologia. 2014 August; 6:703-706. DOI: 